\title{
High expression of progesterone receptor may be an adverse prognostic factor in oestrogen receptor-negative/ progesterone receptor-positive breast cancer: results of comprehensive re-evaluation of multi-institutional case series
}

\author{
Michal Kunc ${ }^{1}$, Rafal Pęrsa ${ }^{1}$, Gabor Cserni ${ }^{2,3}$, Ewa Iżycka-Ś'wieszewska ${ }^{4}$, \\ Aleksandra Łacko ${ }^{5,6}$, Barbara Radecka ${ }^{7,8}$, Marcin Braun ${ }^{9}$, \\ JoAnna Pikiel $^{10}$, Maria Litwiniuk ${ }^{11}$, Katarzyna Pogoda ${ }^{12}$, \\ Anna Szwajkosz ${ }^{13}$, Wojciech Biernat ${ }^{1}$, ElżBieta Senkus ${ }^{14}$ \\ ${ }^{1}$ Department of Pathomorphology, Medical University of Gdańsk, Gdańsk, Poland; \\ ${ }_{3}^{2}$ Department of Pathology, Bács-Kiskun County Teaching Hospital, Kecskemét, Hungary; \\ ${ }^{3}$ Institute of Pathology, University of Szeged, Szeged, Hungary; ${ }^{4}$ Department of Pathology \\ and Neuropathology, Medical University of Gdańsk, Gdańsk, Poland; ${ }^{5}$ Lower Silesian \\ Oncology Centre, Breast Unit, Wroclaw, Poland; ${ }^{6}$ Department of Oncology, Wroctaw \\ Medical University, Wroclaw, Poland; ${ }^{7}$ Department of Oncology, Institute of Medical Sci- \\ ences, University of Opole, Opole, Poland; ${ }^{8}$ Tadeusz Koszarowski Cancer Center, Opole, \\ Poland; ${ }^{9}$ Department of Pathology, Chair of Oncology, Medical University of Łódź, Łódź, \\ Poland; ${ }^{10}$ Regional Oncology Center, Gdynia, Poland; ${ }^{11}$ Greater Poland Cancer Centre, \\ Poznań University of Medical Sciences, Poznań, Poland; ${ }^{12}$ Department of Breast Cancer and \\ Reconstructive Surgery, Maria Sklodowska-Curie National Research Institute of Oncology, \\ Warsaw, Poland; ${ }^{13}$ Oncology Ward, Beskid Oncology Centre-John Paul II Municipal Hos- \\ pital in Bielsko-Biata, Bielsko-Biata, Poland; ${ }^{14}$ Department of Oncology and Radiotherapy, \\ Medical University of Gdańsk, Gdańsk, Poland
}

\section{Summary}

Oestrogen receptor (ER)-negative $(-)$ progesterone receptor (PgR)-positive $(+)$ is the least common combination of steroid receptor expression observed in breast cancer. There are many controversies regarding the actual existence of ER-/PgR+ phenotype. In the current study, we aimed to perform comprehensive immunohistochemical reevaluation of ER-/PgR+ breast cancers from multiple institutions. A total of 135 cases of ER-/PgR+ breast cancer were collected from 11 institutions from the period 2006-2020 and subsequently stained with three clinically validated anti-ER antibody clones: SP1 (Roche), 1D5 (Dako), and EP1 (Dako), and two anti-PgR antibody clones: 636 (Dako), and 1E2 (Roche). Clinicopathological characteristics of confirmed and re-categorised cases were analysed. Seventy-six cases retained the original ER-/ $\mathrm{PgR}+$ phenotype, including $21 \mathrm{HER} 2+$ and $55 \mathrm{HER} 2-$ tumours. Forty-seven cases were ER+ with at least one antiER antibody, and 12 cases were re-categorised as doublenegatives across all anti-ER and anti-PgR antibodies. No significant differences in survival were observed between groups in the HER2+ category. In the HER2- cohort, confirmed ER-/PgR+, ER+ tumours with discrepant ER staining, and triple negatives had inferior overall survival compared to concordant ER+ cases. Progesterone receptor expression in $>20 \%$ of cells was identified as an adverse prognostic factor in ER-/PgR+/HER2- breast cancer in a multivariable model adjusted by stage (HR $5.0,95 \% \mathrm{Cl}$
\end{abstract}

1.3-19.2, $p=0.019)$. We performed one of the largest validation studies so far on $\mathrm{ER}-/ \mathrm{PgR}+$ breast cancer and confirmed the existence of this subgroup. Moreover, we identified high $\mathrm{PgR}$ expression as an adverse prognostic factor.

Key words: Oestrogen receptor; progesterone receptor; breast cancer; immunohistochemistry; prognosis.

Received 4 August, revised 30 September, accepted 11 October 2021 Available online 21 January 2022

\section{INTRODUCTION}

The expression of progesterone receptor $(\mathrm{PgR})$ in breast cancer is induced by oestrogen receptor $\alpha$ (ER). Therefore, the most common phenotype recognised in clinical practice is $\mathrm{ER}+/ \mathrm{PgR}+$ (i.e., double-positive) breast cancer, whereas $\mathrm{ER}-/ \mathrm{PgR}+$ phenotype is very infrequent. Multiple authors have neglected its existence or suggested an artifactual origin. ${ }^{1-4}$ The frequency of ER-/PgR+ tumour diagnosis has decreased in recent years, probably due to improved immunohistochemistry (IHC) techniques. ${ }^{5}$ The recommendations of the American Society of Clinical Oncology/College of American Pathologists (ASCO/CAP) advocate for repeated evaluation of ER expression from alternative blocks to reduce the risk of false negative results. ${ }^{6}$ Nevertheless, even in restrictively controlled laboratories, $\mathrm{ER}-/ \mathrm{PgR}+$ breast cancers are observed and pose a serious challenge for oncologists 
since no specific guidelines address the treatment of ER-/ PgR+ breast cancer. Recently, with RNA sequencing analysis, Beltjens et al. demonstrated that $\mathrm{ER}-/ \mathrm{PgR}+$ breast cancer is molecularly similar to triple negative breast cancer. ${ }^{7}$ Up-regulation of the suppressor of zest 12 (SUZ12) may be a driver of the aggressive phenotype in these tumours. ${ }^{7}$ A few small studies showed they were sensitive to both endocrine treatment and chemotherapy. ${ }^{8,9}$ Moreover, PgR+ status in ER - tumours influences the current American Joint Committee on Cancer (AJCC) staging of breast cancer. ${ }^{10}$

To date, only a few studies have aimed to comprehensively re-evaluate ER-/PgR+ breast cancers. ${ }^{3,4,11-13}$ Unfortunately, their results are contradictory and, at least partially, dependent on the anti-ER antibody used. Only one study analysed single cases of $\mathrm{ER}-/ \mathrm{PgR}+$ breast cancer with three ER assays available for commonly used autostainer vendors (1D5 mixed with ER-2-123 in Dako, 6F11 in Leica, SP1 in Ventana) and demonstrated substantial differences, with no concordant case across all three analysed samples. ${ }^{14}$ In the current retrospective multicentre study, we aim to reappraise diagnoses of $\mathrm{ER}-/ \mathrm{PgR}+$ breast cancers collected from Polish and Hungarian centres, to identify the causes of misdiagnoses, and to analyse the clinical behaviour of any confirmed ER-/PgR+ breast cancers.

\section{MATERIALS AND METHODS}

\section{Study group}

The core group of patients was identified in the medical records of the University Clinical Center in Gdańsk (UCCG) using the MedStream Designer tool in the period from 2006 to 2020. In the other centres, cases were identified in the local databases and sent for central evaluation. Basic clinicopathological data (age, TNM stage, grade, HER2 status, Ki67 expression) and information concerning patients' clinical course (type of treatment, presence of relapse, and/or death) were collected, if available. Finally, 151 cases of $\mathrm{ER}-/ \mathrm{PgR}+$ tumour were collected from nine Polish and two Hungarian centres. Study exclusion criteria comprised cases lacking tissue samples unexposed to systemic therapy ( $n=14)$, cases with hormone receptor (HR) status established only in the nodal or distant metastases $(n=0)$, and lacking invasive component $(n=2)$.

Formalin-fixed paraffin-embedded (FFPE) tissue blocks from 135 preselected cases consisted of 86 core needle or vacuum assisted breast biopsies and 76 post-operative samples. In 27 cases both pre- and post-operative material was available for comparison. The study was approved by the Bioethical Committee of the coordinating centre, the Medical University of Gdansk, Poland (approval no: NKBBN/119/2018). All research was performed in accordance with the appropriate regulations.

\section{Immunohistochemistry}

All cases enrolled in the study (including those re-categorised after evaluation of primary IHC) were subsequently stained with three antibody clones against ER [1D5, Dako, Denmark; EP1 (routinely used in UCCG laboratory), Dako; SP1, Roche, Switzerland], and anti-PgR [clone 636 (routinely used in UCCG laboratory), Dako] according to the manufacturer's guidelines. To save available tissue material only cases with consistent ER- status across three antibody clones and PgR- or equivocal status obtained with anti-PgR 636 clone were additionally stained with anti-PgR 1E2 clone (Roche). The antibodies not routinely used in our laboratory were validated with breast cancer cases with known ER and PgR IHC status before the study. Characteristics of all utilised antibodies are shown in Table 1. If available, more than one tissue block was used to obtain material for staining (average 2.02 blocks/case, range 1-6). Internal positive controls for ER and $\mathrm{PgR}$ expression were identified in all cases (non-neoplastic glandular elements adjacent to cancer). Nuclear staining in $>1 \%$ of cells was considered positive. $^{6}$

\section{mRNA biomarker assessment}

Additionally, in five selected cases that retained $\mathrm{ER}-/ \mathrm{PgR}+$ status after all steps of re-evaluation, an ESR1/PGR/ERBB2/MKi67 mRNA biomarker assessment was performed to objectify the findings using Xpert Breast Cancer STRAT4 (Cepheid, USA). Briefly, this system is a multiplexed reverse transcription quantitative real-time polymerase chain reaction (RTqPCR) for the quantitative assessment of four breast cancer biomarkers utilising the single use cartridges. FFPE tissue blocks containing cancer tissue were cut into four $20 \mu \mathrm{m}$ slices and then a lysate was prepared using an FFPE Lysis Kit (Cepheid), added to the cartridge, and placed into the GX instrument.

\section{Statistics}

Categorical variables were compared by Fisher's exact test or Chi-square test with Yates correction. The normal distribution of the data was assessed using the Shapiro-Wilk test. Continuous variables were analysed utilising the Mann-Whitney U test or Kruskal-Wallis test when applicable. The agreement between clones of anti-ER antibody was assessed by Fleiss' kappa and Cohen's kappa coefficients.

Overall survival (OS) was defined as the time from the diagnosis to the date of death from any cause. Kaplan-Meier curves were plotted to calculate the survival rates of $\mathrm{ER}-/ \mathrm{PgR}+$ and other phenotypes. Hazard ratios (HRs) were estimated for the different phenotype groups with reference to $\mathrm{ER}+/ \mathrm{PgR}+$ using Cox regression analysis. A $p$ value $<0.05$ was considered significant; in cases of multiple comparisons $p$ values were adjusted at a false discovery rate $(\mathrm{FDR})=0.05$ using Benjamini-Hochberg correction.

Statistical analyses were performed with the use of Statistica 13 (RRID:SCR_014213, Tibco, CA, USA) licensed to Medical University of Gdańsk, and $\mathrm{R}$ statistical environment. ${ }^{15}$ Boxplots and scatterplots were generated using the 'ggplot2' package. ${ }^{16}$ Kaplan-Meier curves were plotted using the 'survminer' and 'ggsci' packages. ${ }^{17,18}$

\section{RESULTS}

\section{Immunohistochemistry}

The detailed characterisation of the study group is shown in Table 2 and the study flowchart is shown in Fig. 1. After primary evaluation, the total number of 135 breast cancer cases primarily designated as $\mathrm{ER}-/ \mathrm{PgR}+$ were enrolled in the IHC study. Eventually, within this group, 76 cases $(56.3 \%)$ retained the original phenotype, in $47(34.8 \%)$ the status was

Table 1 Basic characteristics of anti-ER and anti-PgR antibodies used in the study

\begin{tabular}{|c|c|c|c|c|c|c|}
\hline Antigen & Antibody & Manufacturer & Host & Clonality & Autostainer & Concentration \\
\hline ER & 1D5 & Dako & Mouse & Monoclonal & Dako & Ready-to-use \\
\hline ER & EP1 & Dako & Rabbit & Monoclonal & Dako & Ready-to-use \\
\hline PgR & 636 & Dako & Mouse & Monoclonal & Dako & Ready-to-use \\
\hline PgR & $1 \mathrm{E} 2$ & Roche & Rabbit & Monoclonal & Ventana & Ready-to-use \\
\hline
\end{tabular}


Table 2 The clinicopathological characteristic of the study group

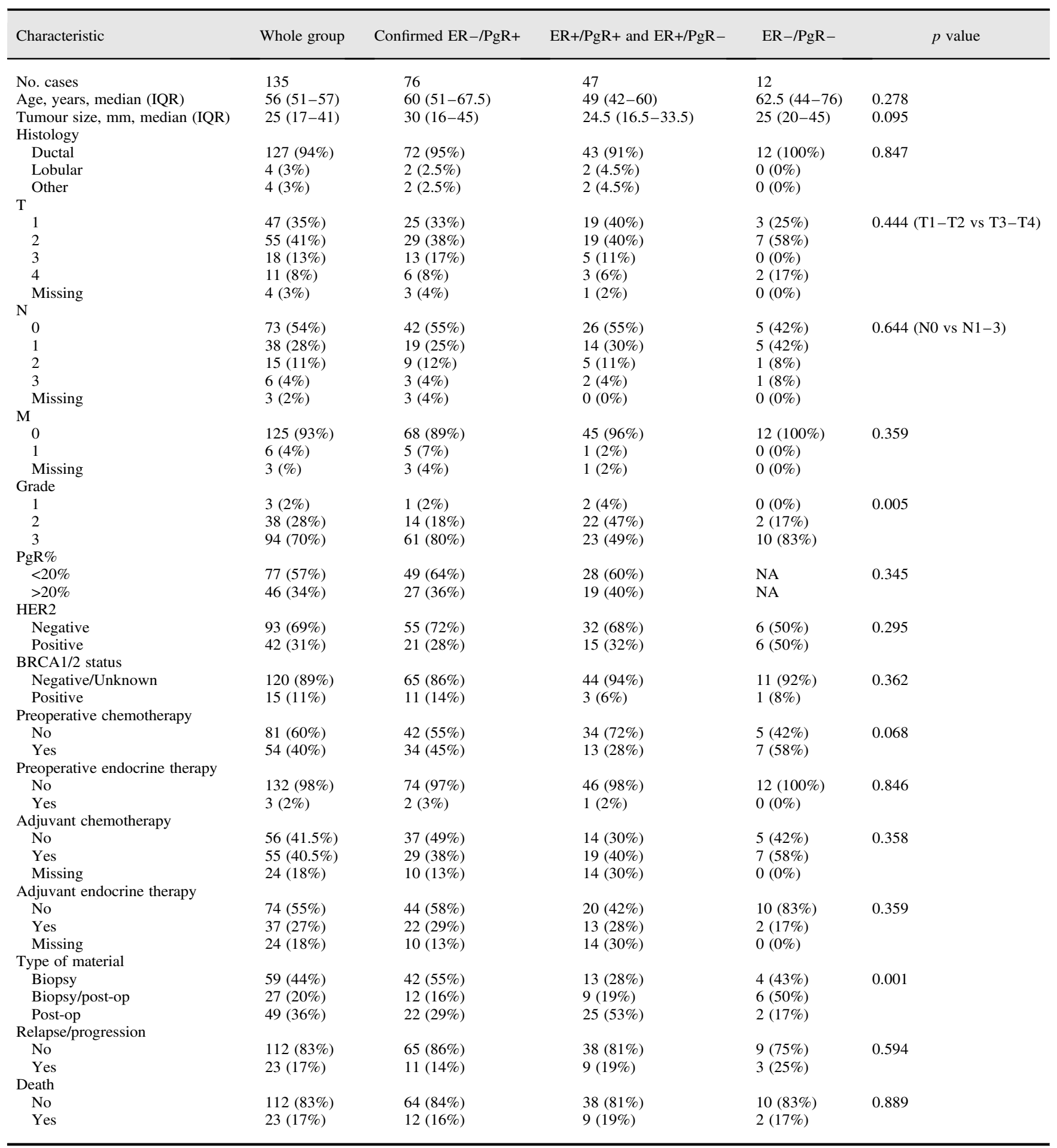

$p$ value calculated with chi square test.

$\mathrm{ER}$, oestrogen receptor; IQR, interquartile range; NA, not applicable; PgR, progesterone receptor.

changed to ER+, and $12(8.9 \%)$ tumours were doublenegatives. The examples of confirmed ER-/PgR+ breast cancer are shown in Fig. 2 and Fig. 3.

Discordant expression of ER in various tissue blocks occurred in three cases $(2.2 \%)$, leading to the change of a phenotype to $\mathrm{ER}+/ \mathrm{PgR}+$. This may suggest that tumour heterogeneity is rarely accountable for a single hormone receptor phenotype. It is further supported by the finding that in all confirmed ER $-/ \mathrm{PgR}+$ breast cancers with core needle biopsy and chemo-naïve resection specimen available for comparison, the results were concordant. Additionally, we compared the group with only one FFPE tissue block available and the group with $>1$ block analysed, and no statistically significant difference between these groups was noted in the frequency of confirmed ER-/PgR+ cases $(63 \%$ vs $48.5 \%, p=0.138$, chi-square). 


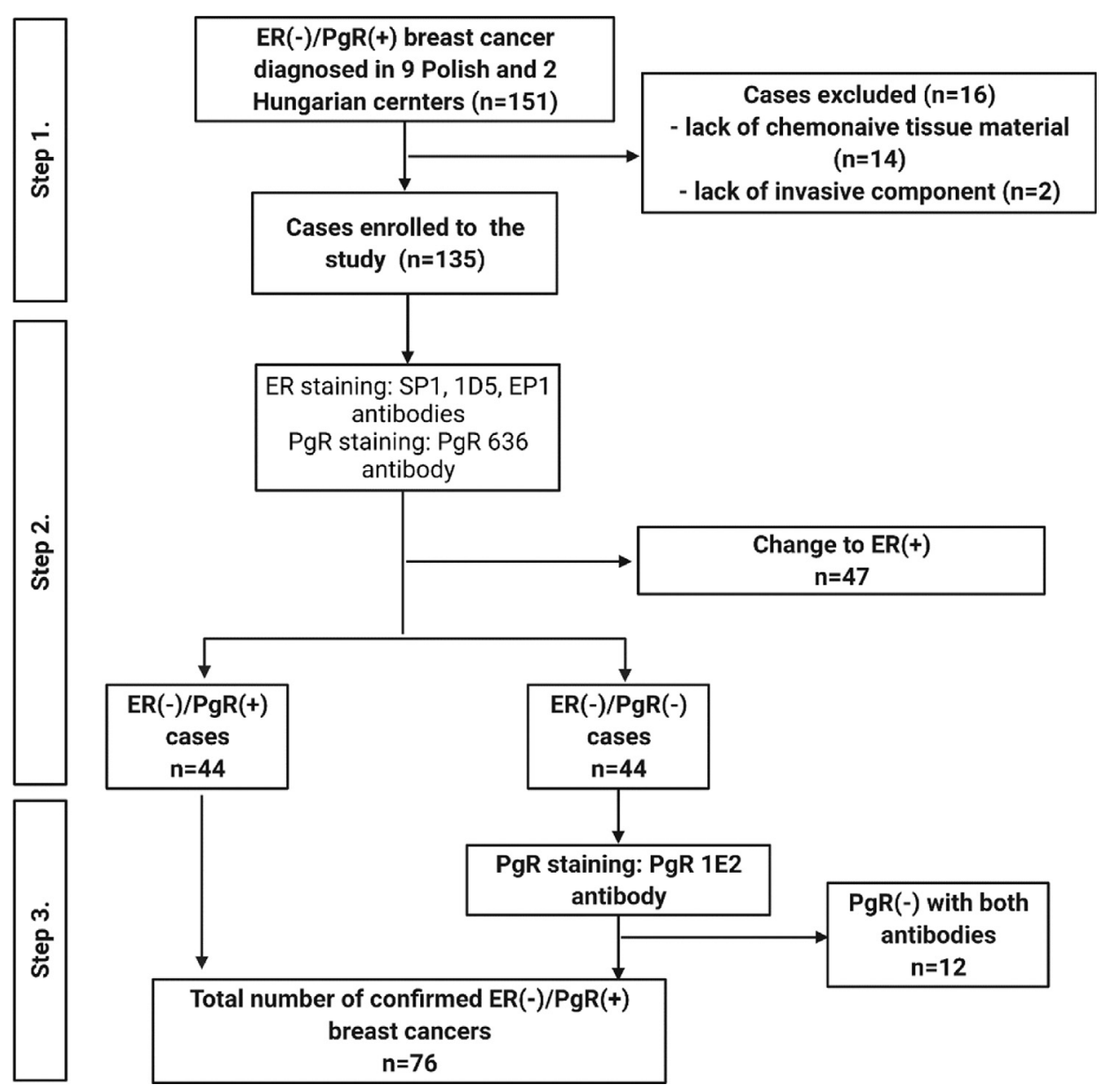

Fig. 1 Flowchart of the study.

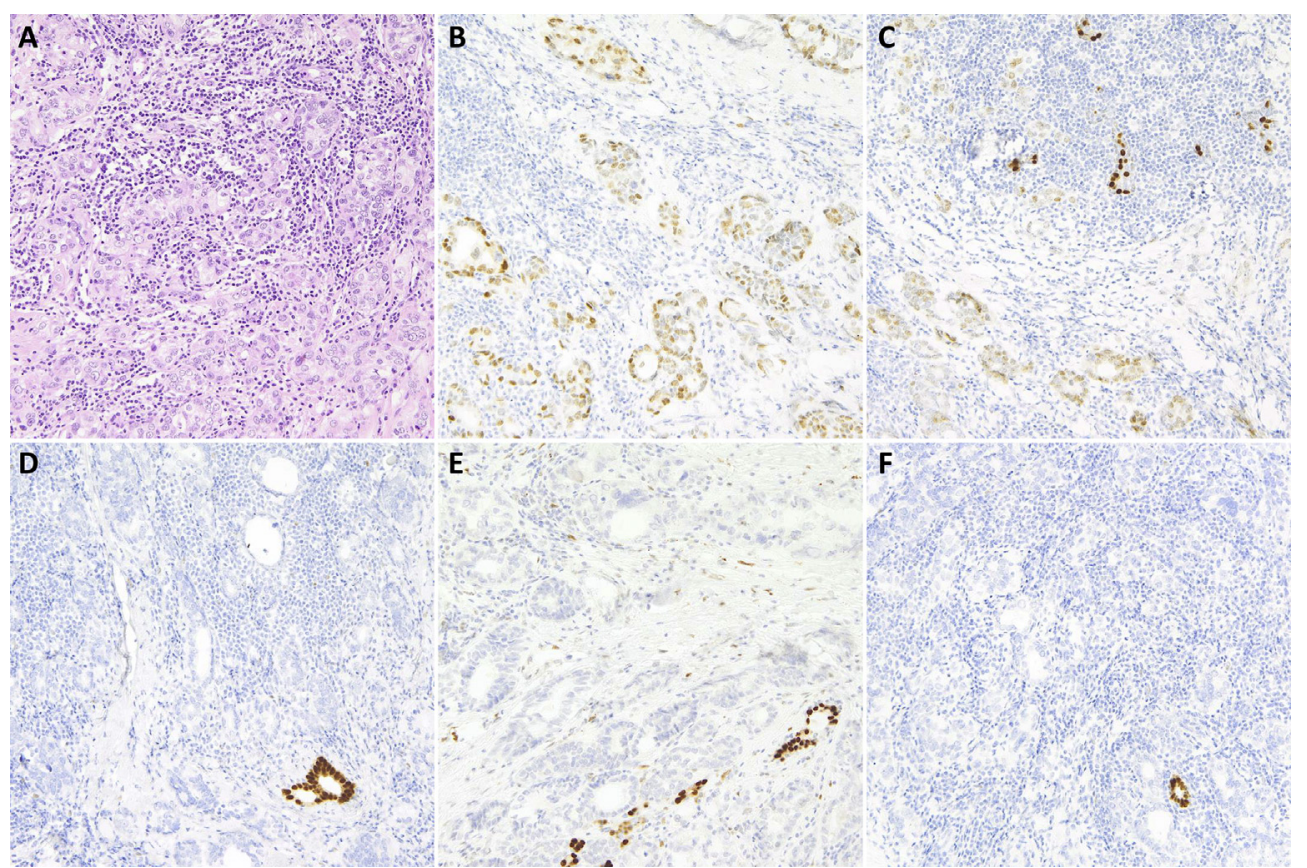

Fig. 2 Example of confirmed ER-/PgR+ breast cancer. (A) Neoplastic tubules scattered in the lymphocyte-rich stroma in haematoxylin and eosin staining; (B) positive PgR 636 staining with (C) positive control; negative ER stainings with positive internal controls, (D) EP1, (E) SP1, (F) 1D5. ER, oestrogen receptor; PgR, progesterone receptor. 


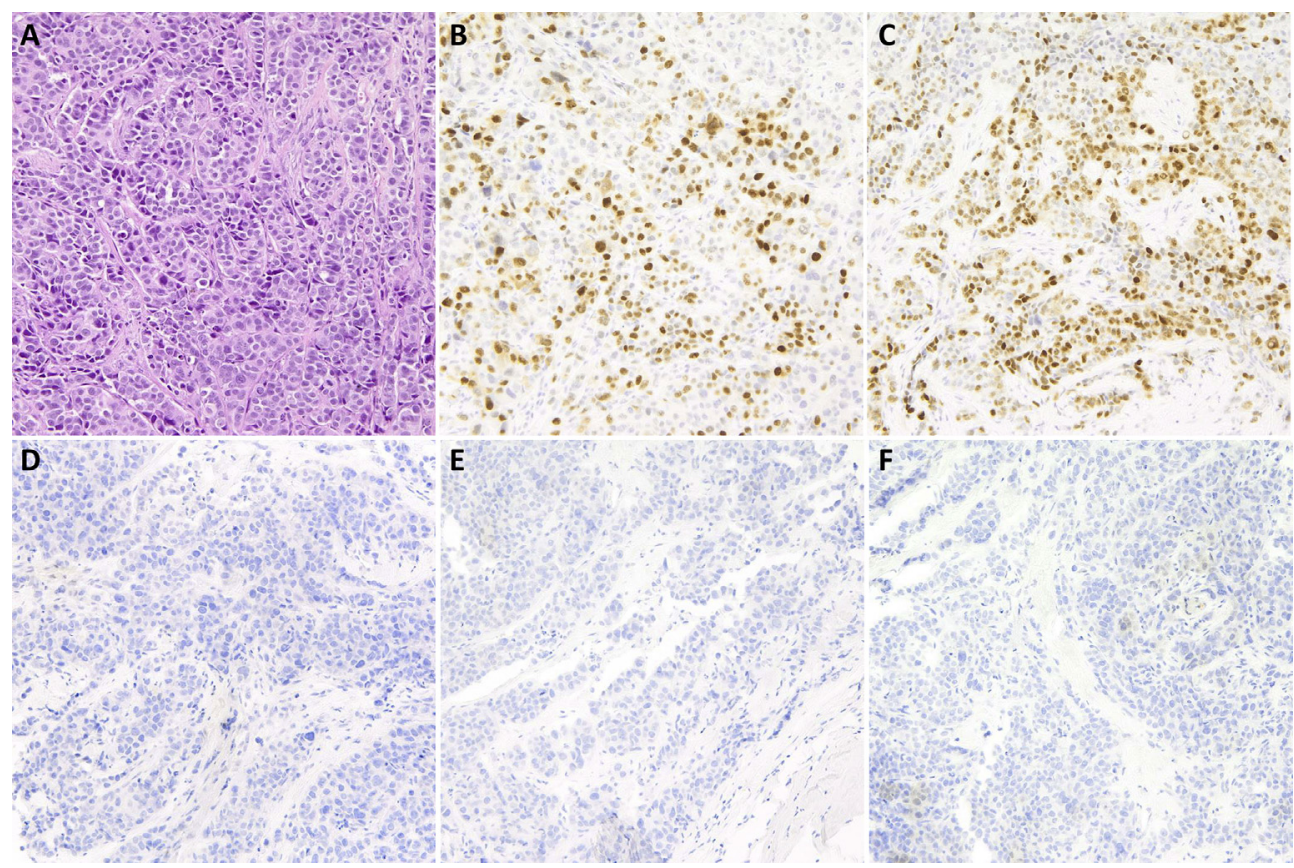

Fig. 3 Example of confirmed ER-/PgR+ breast cancer. (A) Core needle biopsy of high grade infiltrating duct carcinoma in haematoxylin and eosin staining; (B,C) positive PgR 636 staining; negative ER stainings with (D) EP1, (E) SP1, (F) 1D5 (F). Internal positive control for ER was detected in this specimen with all clones. ER, oestrogen receptor; $\mathrm{PgR}$, progesterone receptor.

We observed non-negligible discrepancies in results of ER staining, but the general agreement in binary classification into negative or positive expression across three investigated clones was substantial (Fleiss' kappa 0.73). The worst concordance was observed between the SP1 and EP1 clones (Table 3). Similarly, a correlation between the percentage of positively staining nuclei was high between all three antibodies, but the correlation was the weakest for SP1 and EP1 (Fig. 4). Overall, discordant stainings were present in $21(15.5 \%)$ of tumours. Positive concordance across three antibody clones was observed in $26(19.3 \%)$, and negative in $88(65.18 \%)$ cases.

We also observed a discrepancy in PgR staining. Of 42 PgR - cases by 636 clone, staining with 1E2 clone demonstrated positive nuclear reaction in 32 (76.2\%). Eventually, 76 tumours showed concordant ER- phenotype with three anti-ER antibody clones and PgR expression in at least one anti-PgR clone. The examples of discrepant ER and PgR stainings are shown in Fig. 5.

Further proof of the existence of the $\mathrm{ER}-/ \mathrm{PgR}+$ phenotype was obtained at the molecular level. Xpert Breast Cancer STRAT4 confirmed the ER $-/ \mathrm{PgR}+$ phenotype in four of five

Table 3 The concordance between anti-ER antibodies in the binary classification of ER expression (negative versus positive)

\begin{tabular}{llll}
\hline & ER 1D5+ & ER 1D5- & Cohen's kappa (95\% CI) \\
\hline ER SP1+ & 33 & 12 & $0.786(0.673-0.899)$ \\
ER SP1 & 0 & 90 & \\
\hline & ER EP1+ & ER EP1- \\
\hline ER SP1+ & 26 & 19 & $0.613(0.470-0.757)$ \\
ER SP1 - & 2 & 88 & \\
\hline & ER 1D5+ & ER 1D5- \\
\hline ER EP1+ & 26 & 2 & $0.810(0.691-0.929)$ \\
ER EP1- & 7 & 100 & \\
\hline
\end{tabular}

cases in which the analysis was performed (Fig. 6). A single case unconfirmed in mRNA assay (ER/PgR negative in the mRNA analysis) had $15 \%$ of PgR+ nuclei in IHC staining.

\section{Clinicopathological features of confirmed ER-/PgR+ cases}

The vast majority of confirmed ER-/PgR+ cases ( $n=61$, $80.3 \%$ ) were classified as grade 3 , and almost all of them $(n=72,94.7 \%)$ exhibited morphology of infiltrating duct carcinoma of no special type (Table 2). HER2 overexpression or amplification was detected in 21 tumours $(27.6 \%)$. The highest percentage of $\mathrm{PgR}+$ cells was observed in ER+/ PgR+/HER2 - tumours, whereas ER-/PgR+/HER2 - and discordant $\mathrm{ER}+/ \mathrm{PgR}+/ \mathrm{HER} 2$ - breast cancers were characterised by significantly lower percentage of $\mathrm{PgR}+$ cells (Fig. 7A). No significant differences in the percentage of $\mathrm{PgR}+$ cells were noted in the HER2+ group but the number of cases in this subgroups was low (Fig. 7B).

Within the confirmed ER-/PgR+ subgroup, patients most frequently presented with T2 tumours $(n=29,38.2 \%)$. Nodal metastases were observed in 31 patients $(40.8 \%)$, and five patients $(6.6 \%)$ manifested de novo distant metastatic disease. Almost all tumours with available Ki67 results displayed a very high proliferation index (median 60\%). Thirty-four $(44.7 \%)$ patients received pre-operative chemotherapy, and 12 tumours $(35.3 \%)$ achieved complete pathological response (pCR). The response rate was not statistically different from re-categorised (ER+ and/or PgR-) groups.

In the whole cohort, 37 patients were treated with adjuvant endocrine therapy $(27.4 \%)$, including 22 patients $(28.9 \%)$ with confirmed ER-/PgR+ diagnoses. Adjuvant chemotherapy was administered to 55 patients $(40.7 \%)$ in the whole cohort, including 29 confirmed ER-/PgR+ cases $(38.2 \%)$. Pre-operative and post-operative anti-HER2 treatment was administered to nine patients $(6.7 \%)$ and 28 patients $(20.74 \%)$, respectively. In the confirmed ER-/PgR+/HER2+ group, anti- 

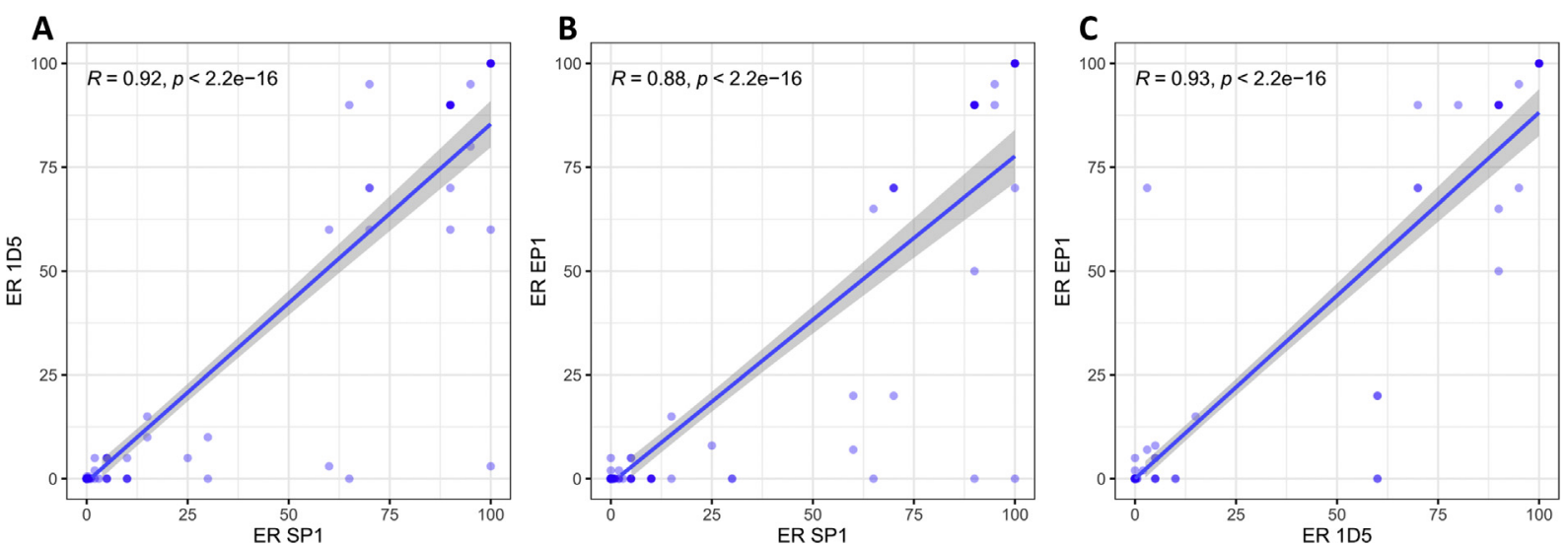

Fig. 4 Correlation between percentage of positively staining cells between three clones of anti-ER antibody. ER, oestrogen receptor; R, Spearman's rank correlation coefficient.

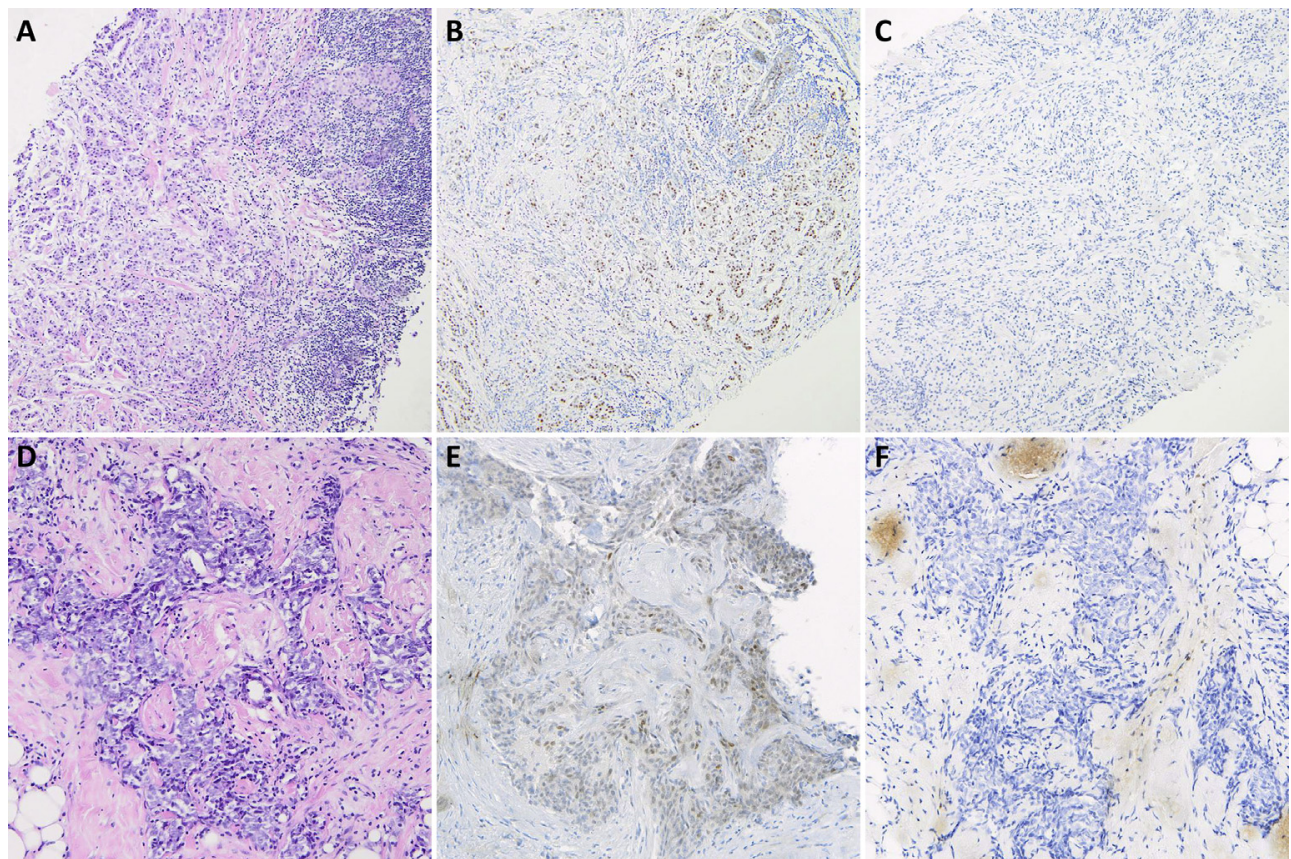

Fig. 5 Examples of discrepant ER and PgR stainings. (A-C) First case: (A) haematoxylin \& eosin staining, (B) positive PgR 1E2 staining, and (C) negative PgR 636 staining. (D-F) Second case: (D) haematoxylin \& eosin staining, (E) weakly positive ER SP1 staining, and (F) negative ER EP1 staining. ER, oestrogen receptor; PgR, progesterone receptor.

HER2 treatment was applied in the neoadjuvant setting to 5/21 patients $(23.8 \%)$, and post-operatively in $15 / 21$ patients (71.4\%; information was missing for 3 patients, $14.3 \%$ ).

\section{Survival of confirmed ER-/PgR+ breast cancer compared to re-categorised cases}

The OS was $81.3 \%$ in the whole group with a median followup time of 44 months [interquartile range (IQR) 27-59 months). To assess clinical consequences of $\mathrm{ER}-/ \mathrm{PgR}+$ breast cancer reclassification we performed survival analyses comparing confirmed and recategorised cases.

In the group of HER2 - breast cancer, the best outcomes were observed in patients with ER+ status confirmed by three anti-ER antibodies (Fig. 8A). Confirmed ER-/PgR+ cases, triple-negative cancers, and ER+ tumours with discordant staining results (lacking agreement across all anti-ER clones) had a similar clinical course with inferior outcomes (Table 4).
In the group of HER2+ tumours we did not observe any significant differences in survival, but a smaller number of cases might have influenced the results, and precluded calculation of HRs. Nevertheless, we observed a trend toward better prognosis in HER2-overexpressing/amplified ER-/ $\mathrm{PgR}+$ breast cancer when compared to ER-/PgR+/HER2cases with borderline statistical significance (log-rank $p=0.067 ; \mathrm{HR}=0.20,95 \%$ confidence interval $0.03-1.605$ ) (Fig. 8B).

Regarding treatment modalities, in the group of confirmed $\mathrm{ER}+/ \mathrm{PgR}$ - cases, we did not find any statistically significant effects on OS taking into consideration pre-operative chemotherapy $(\mathrm{HR}=0.98,95 \%$ CI $0.31-3.11, p=0.97)$, post-operative chemotherapy ( $\mathrm{HR}=0.90,95 \%$ CI $0.26-3.13$, $p=0.874)$, and post-operative endocrine therapy $(\mathrm{HR}=0.44$, $95 \%$ CI $0.09-2.14, p=0.312$ ). No death was noted amongst patients treated with anti-HER2 drugs in adjuvant setting (HR impossible to calculate). 
A

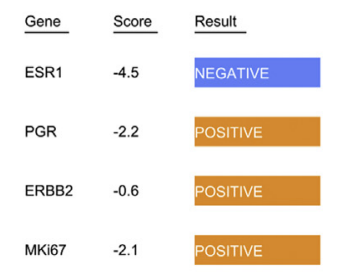

C

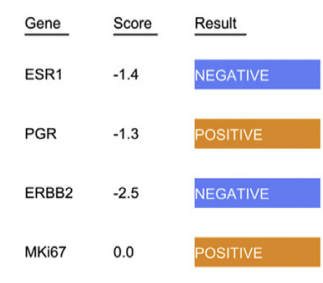

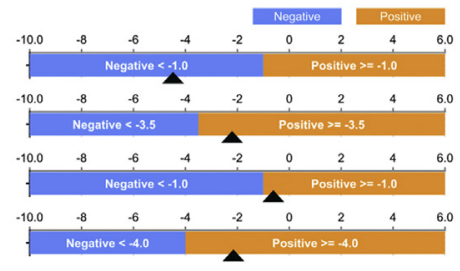

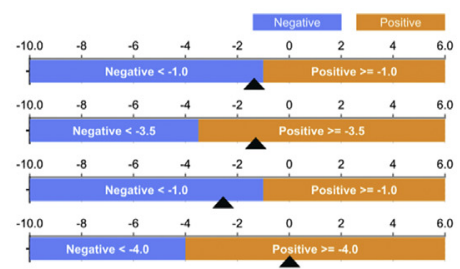

E

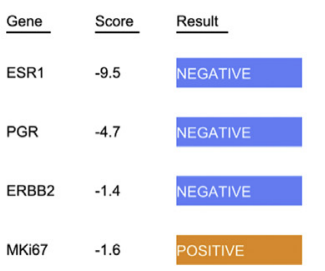

B
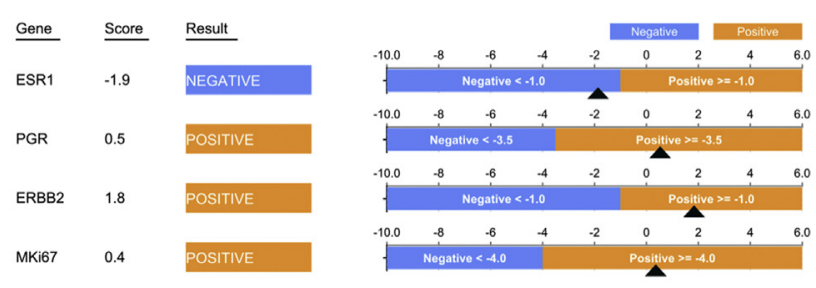

$\mathrm{D}$
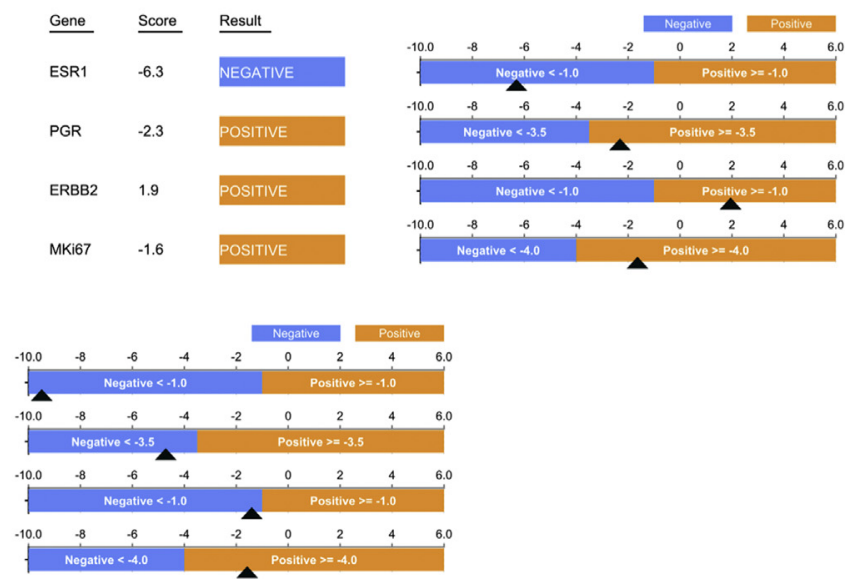

Fig. 6 (A-E) The results of Xpert Breast Cancer STRAT4 assay in five ER-/PoR+ breast cancer cases confirmed by immunohistochemistry. (E) The case negative in STRAT4 assay immunohistochemistry revealed positive PgR staining in 15\% of nuclei. ERBB2, Erb-B2 receptor tyrosine kinase 2; ESR1, oestrogen receptor 1; MKi67, marker of proliferation Ki-67; PGR, progesterone receptor.
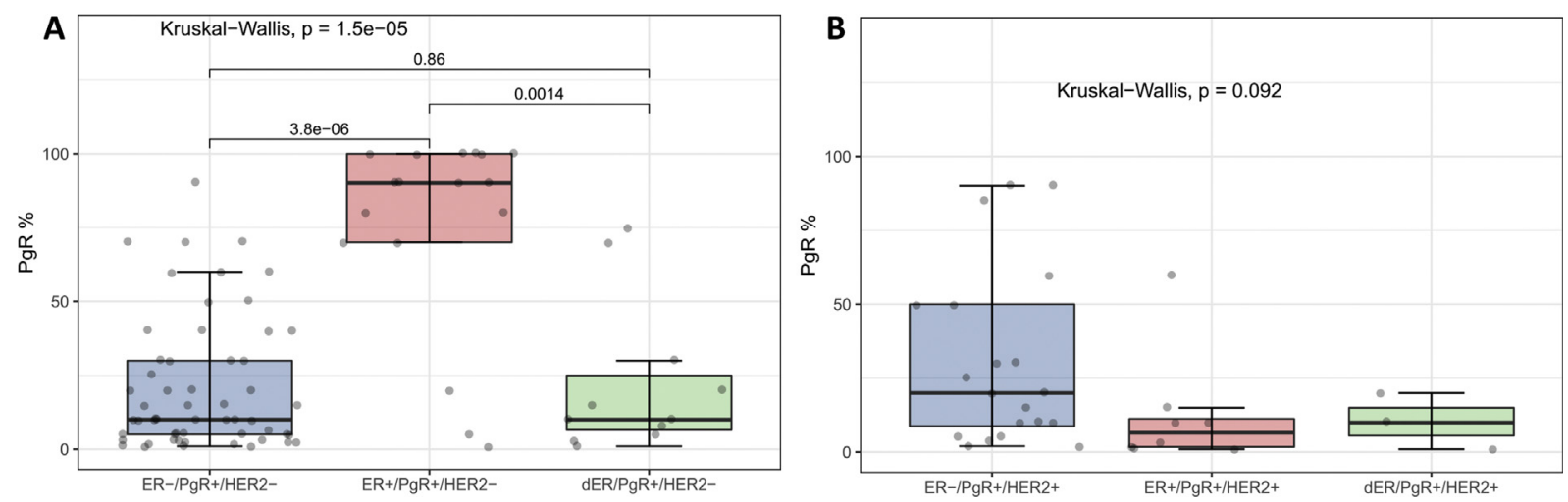

Fig. 7 Percentage of PgR+ cells according to the phenotype of (A) HER2 - and (B) HER2+ breast cancer. The boxplot displays the median values of the percentage of $\mathrm{PgR}+$ cells, which is indicated by the centreline with a dot. The edges of the boxes represent the 25 th percentile and 75 th percentile. The horizontal lines outside the boxes show the maximum and minimum values of the data that are within 1.5 times the interquartile range over the 75 th and 25 th percentile, respectively. Dots depict individual measures. D, discordant staining; ER, oestrogen receptor; PgR, progesterone receptor.

In the next step, we assessed the prognostic significance of the PgR expression level on survival among $\mathrm{ER}-/ \mathrm{PgR}+/$ HER2 - tumours. The receiver operating curve was plotted to find the cut-off value discriminating patients in terms of fatal outcomes (Fig. 9). Surprisingly, breast cancers expressing PgR in $>20 \%$ of cells showed inferior OS (Fig. 10A). This effect was retained utilising either local or central assessment of the $\mathrm{PgR}$ expression. Moreover, there was no difference in OS between PgR 636+ and PgR 1E2+ only tumours. As the stage is the crucial prognostic factor in breast cancer, we evaluated the prognostic impact of the percentage of PgR-expressing cancer cells in the multivariable Cox regression model adjusted by stage (Table 5). The status of PgR retained its statistical significance in multivariate analysis. The opposite trend (without statistical significance) was observed in ER+/ $\mathrm{PgR}+$ group, in which a higher percentage of $\mathrm{PgR}+$ cells was associated with longer OS (Fig. 10B).

\section{DISCUSSION}

$\mathrm{ER}-/ \mathrm{PgR}+$ breast cancer is the most controversial breast cancer subtype defined by hormone receptor status. This is the first approach to assess these cancers in Poland. In Hungary, a previous study confirmed the ER-/PgR+ phenotype in only one case from a cohort of 182 cases. $^{12}$

The frequency of $\mathrm{ER}-/ \mathrm{PgR}+$ cancer diagnosis has decreased in recent years and stabilised in most countries at the level of approximately $1.0-1.5 \%$. According to the data 

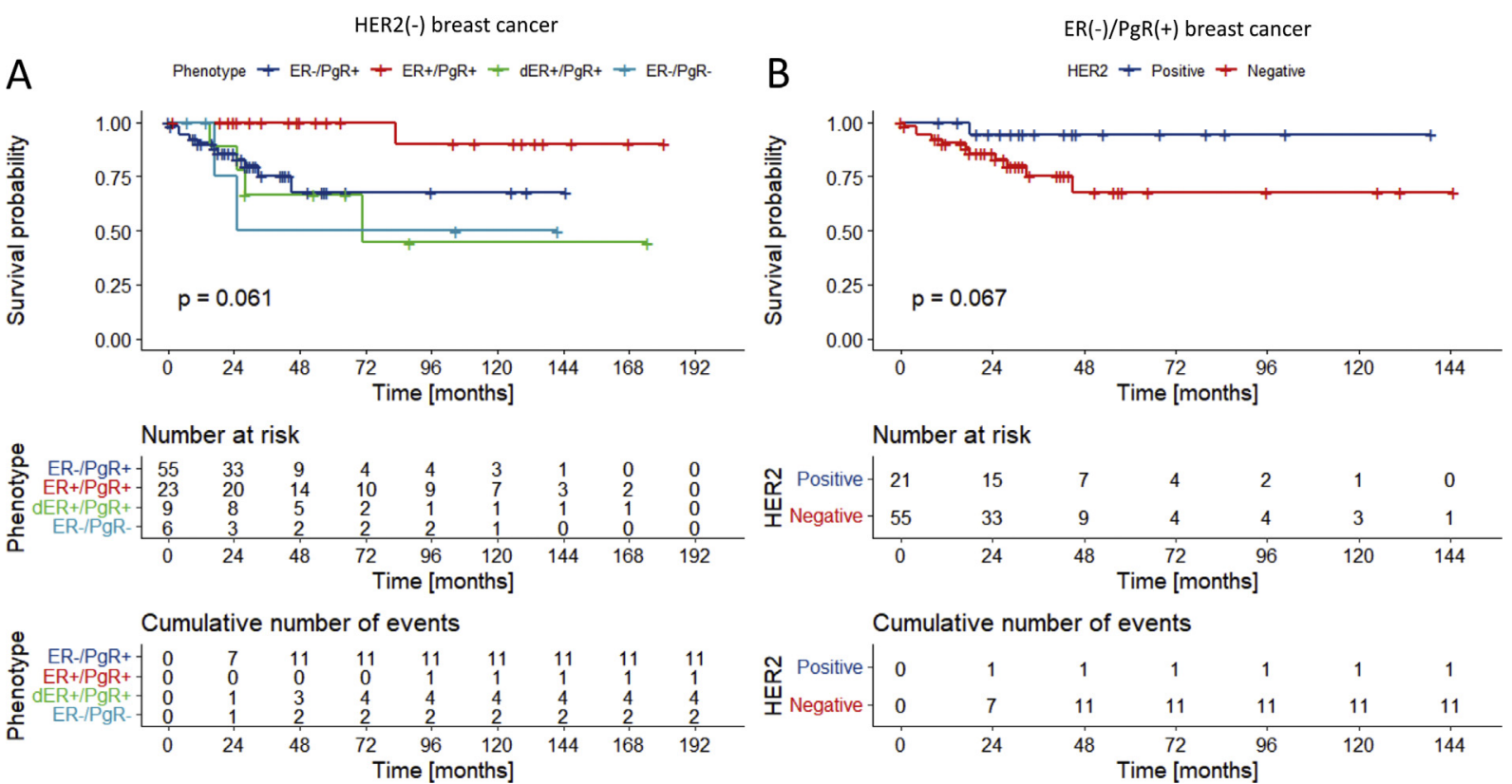

Cumulative number of events

\begin{tabular}{|c|c|c|c|c|c|c|c|}
\hline \multirow{3}{*}{ 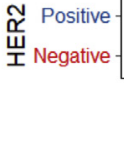 } & 0 & 1 & 1 & 1 & 1 & 1 & 1 \\
\hline & 0 & 7 & 11 & 11 & 11 & 11 & 11 \\
\hline & 0 & 24 & 48 & $\begin{array}{l}72 \\
e[1\end{array}$ & 96 & 120 & 144 \\
\hline
\end{tabular}

Fig. 8 (A) Overall survival of HER2 - breast cancers divided by ER and PgR status, including discordant ER+ group; (B) overall survival of ER-/PgR+ breast cancer stratified by HER2 status. Demonstrated $p$ values were calculated with a log-rank test. D, discordant staining; ER, oestrogen receptor; PgR, progesterone receptor.

Table 4 The results of univariate Cox proportional hazard analysis in HER2 - breast cancer in reference to ER+/PgR+ group

\begin{tabular}{llllll}
\hline Phenotype & HR & 95\% CI & $p$ (Cox) & Adjusted $p$ (Cox) & $p$ (log-rank) \\
& & & Adjusted $p$ (log-rank) \\
\hline ER+ with concordant results & 1.000 & N/A & N/A & N/A & N/A \\
ER+ with discordant results & 12.343 & $1.357-112.19$ & 0.025 & 0.075 & 0.006 \\
ER-/PgR+ & 8.314 & $1.035-66.77$ & 0.046 & 0.069 & 0.015 \\
ER-/PgR- & 11.186 & $1.012-123.680$ & 0.048 & 0.048 & 0.022 \\
& & & & 0.024 \\
\hline
\end{tabular}

Adjusted $p$ values were calculated with Benjamini-Hochberg correction.

$\mathrm{CI}$, confidence interval; ER, oestrogen receptor; HR, hazard ratio; PgR, progesterone receptor.

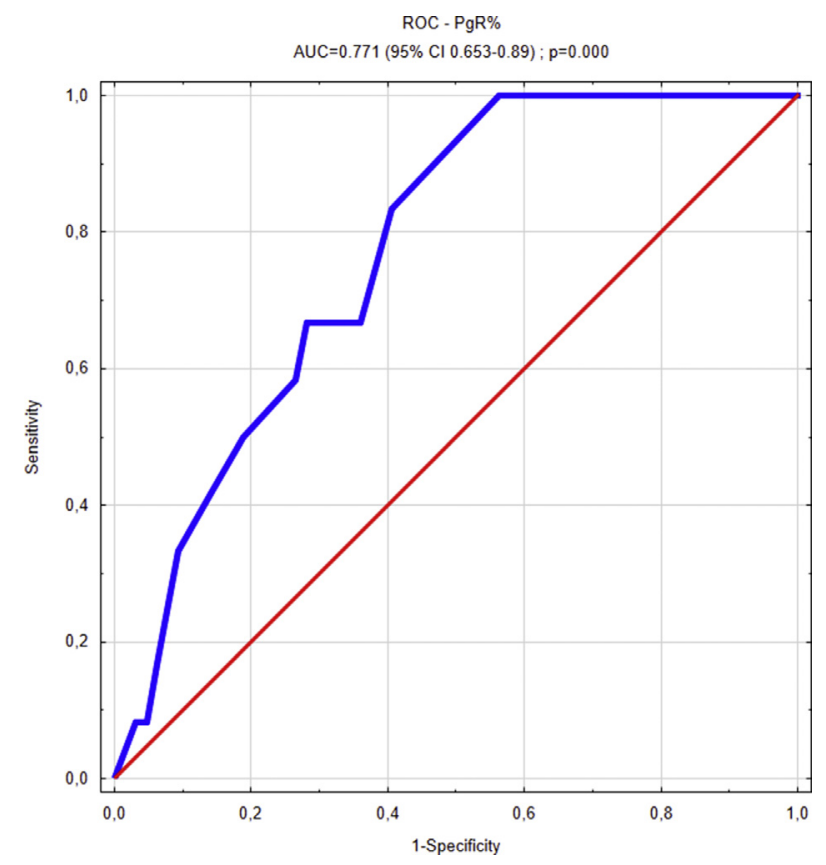

Fig. 9 Receiver operating curve for the percentage of PgR+ cancer cells plotted against death. AUC, area under curve; CI, confidence interval; ROC, receiver operating curve. from Surveillance, Epidemiology, and End Results (SEER) in the USA, the drop in ER-/PgR+ phenotype has been noted since $1989 .{ }^{19}$ These changes are believed to be associated with the switch from the ligand-binding assay to IHC and subsequent improvements in IHC detection methods. Moreover, the threshold for ER positivity was reduced from $10 \%$ to $1 \%$. Some authors postulate that $\mathrm{ER}-/ \mathrm{PgR}+$ tumours should not be diagnosed if any nuclear staining for ER in cancer cells is present. ${ }^{2,4}$ Hereby, we utilised $1 \%$ of reactive nuclei as a threshold, which is recommended by the current guidelines, and validated in clinical practice. ${ }^{6}$

We identified several possible causes of $\mathrm{ER}-/ \mathrm{PgR}+$ misdiagnosis. One of the avoidable mistakes is a typo or mislabelling of the phenotype in the pathology report, e.g., designation of $\mathrm{ER}+/ \mathrm{PgR}$ - tumour as $\mathrm{ER}-/ \mathrm{PgR}+$. Another possibility is a misinterpretation of cytoplasmic PgR expression as positive, counting entrapped non-neoplastic glandular $\mathrm{PgR}+$ cells as cancer cells, and $\mathrm{PgR}$ expression in $<1 \%$ of cells. In several cases, we observed weak and focal expression of PgR in stromal cells or immune cells. Finally, weak nuclear expression of ER in a low number of cells (1-10\%) might have gone unnoticed in some cases or have been confused with non-neoplastic glands in well-differentiated tumours.

In two recent studies, ER-/PgR+ cancers showed worse prognosis than double-positive and $\mathrm{ER}+/ \mathrm{PgR}$ - tumours, 

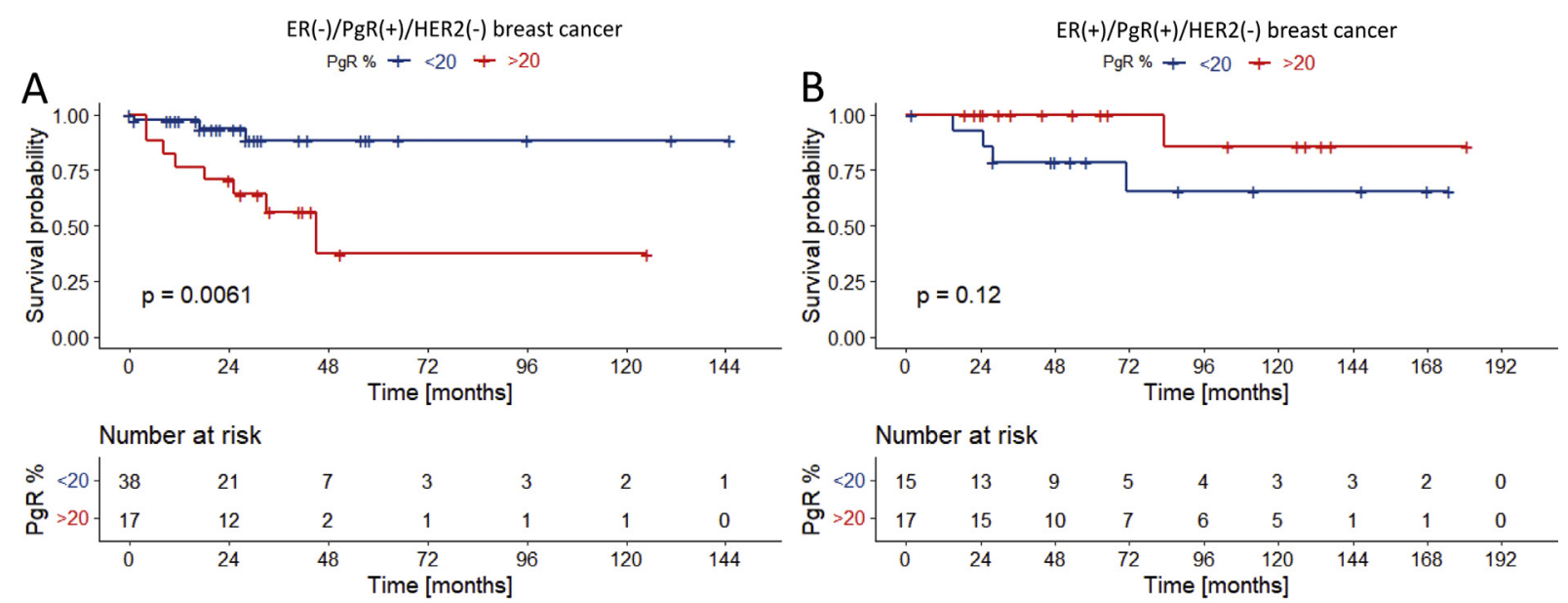

Cumulative number of events

\begin{tabular}{|c|c|c|c|c|c|c|c|}
\hline \multirow{3}{*}{$\begin{array}{l}\stackrel{\circ}{\alpha}<20 . \\
\underline{\alpha} 0>20 .\end{array}$} & 0 & 2 & 3 & 3 & 3 & 3 & 3 \\
\hline & 0 & 5 & 8 & 8 & 8 & 8 & 8 \\
\hline & 0 & 24 & 48 & $\begin{array}{c}72 \\
\text { e [m }\end{array}$ & 96 & 120 & 144 \\
\hline
\end{tabular}

Cumulative number of events

\begin{tabular}{|c|c|c|c|c|c|c|c|c|c|}
\hline \multirow{3}{*}{ 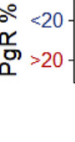 } & 0 & 1 & 3 & 4 & 4 & 4 & 4 & 4 & 4 \\
\hline & 0 & 0 & 0 & 0 & 1 & 1 & 1 & 1 & 1 \\
\hline & 0 & 24 & 48 & 72 & $\begin{array}{l}96 \\
e[m\end{array}$ & $\begin{array}{l}120 \\
\text { ths] }\end{array}$ & 144 & 168 & 192 \\
\hline
\end{tabular}

Fig. 10 (A) Overall survival of ER-/PgR+/HER2 - breast cancer stratified by the percentage of PgR+ cells; (B) overall survival of ER+/HER2 - breast cancer stratified by the percentage of PgR+ cells. Demonstrated $p$ values were calculated with a log-rank test. ER, oestrogen receptor; PgR, progesterone receptor.

Table 5 Multivariable Cox regression model predicting survival in ER-/ $\mathrm{PgR}+/ \mathrm{HER} 2$ - breast cancer including stage and the percentage of PgR+ cells

\begin{tabular}{llll}
\hline Feature & HR & $95 \%$ CI & $p$ \\
\hline Stage $(3-4$ vs $1-2)$ & 3.8 & $1.1-12.9$ & 0.033 \\
PgR $(>20 \%+$ cells vs $<20 \%+$ cells $)$ & 5.00 & $1.3-19.2$ & 0.019 \\
\hline
\end{tabular}

CI, confidence interval; ER, oestrogen receptor; HR, hazard ratio; PgR, progesterone receptor.

which emphasises their distinctive aggressive biology. ${ }^{20,21}$ Our results are consistent with these findings. Thus, we conclude that the tumours with positive ER staining in $<1 \%$ of cells and $\mathrm{PgR}+$ in $>1 \%$ of cells should not be considered double-positive cases, because of their distinctively poor outcomes. Moreover, the majority of these tumours present basal gene expression profiles in the PAM50 classifier, which coincides with their aggressive biology. ${ }^{22}$ One of the most counterintuitive findings in our study was the association between higher expression of $\mathrm{PgR}$ and worse $\mathrm{OS}$ in $\mathrm{ER}-/ \mathrm{PgR}+/$ HER2 - breast cancers. We hypothesise that in the absence of ER expression, PgR may drive the expression of a distinct subset of genes responsible for more aggressive biology. Alternatively, these cancers may display an imbalance in PgR isoforms leading to overexpression of PgR-A isoform, which characterises breast cancers with loss of endocrine sensitivity and poorer outcomes. ${ }^{23,24}$ On the other hand, the trend towards a better prognosis in HER2 + cases is consistent with the recent study based on the SEER database. ${ }^{25}$ This effect is most likely associated with benefits from trastuzumab treatment.

The higher sensitivity of the SP1 antibody compared to the 1D5 antibody is consistent with previous reports. ${ }^{26,27}$ In the study comparing SP1, 6F11, and 1D5 antibodies, nine 1D5negative cases demonstrated weak positivity for SP1 and/or $6 \mathrm{~F} 11 .{ }^{27}$ Of note, these cancers were characterised by high grade, high Ki67 index, HER2 overexpression, and PgR negativity or low positivity, suggesting endocrine resistance. ${ }^{27}$
Another study demonstrated that EP1 antibody has a high concordance with Dako ER/PR pharmDx kit and SP1 antibody, but the use of EP1 improved interpretation of ER IHC results. $^{28,29}$

The very high percentage of $\mathrm{PgR} 636-$, but $1 \mathrm{E} 2+$ cases is one more striking finding in our study. Troxell et al. reported 1E2 antibody generating more positive results in cases generally negative for PgR with other antibody clones. ${ }^{30}$ As emphasised by Kornaga et al., the Roche/Ventana assay recognises both isoforms of PgR, which may explain this discrepancy. ${ }^{31}$ Moreover, the same study suggests that Roche/Ventana assay with 1E2 clone may have superior prognostic value compared to other vendors. ${ }^{31}$ Nevertheless, we accept the possibility that some cases may represent false-positive staining.

Our findings suggest that a diagnosis of $\mathrm{ER}-/ \mathrm{PgR}+$ breast cancer is frequently associated with high grade, low PgR positivity, and high Ki67 index. These findings are supported by the recent study of ER-/PgR+ tumours from Japan, ${ }^{13}$ which concluded with a recommendation for retesting in particular low grade cases, and those with a high proportion of PgR positive cells. Special caution should be applied to biopsy specimens, which may represent a relatively small cancer area dominated by $\mathrm{ER}-/ \mathrm{PgR}+$ cells; however, in our cohort full concordance was present between biopsy and post-operative material amongst confirmed $\mathrm{ER}-/ \mathrm{PgR}+$ cases. Nevertheless, this problem is very difficult to overcome, due to the more and more frequent use of pre-operative systemic therapy, which may lead to complete tumour regression and lack of tissue material for comparison with a biopsy sample.

Our study has several limitations. Most importantly, the cohort was clinically heterogeneous and relatively small, which is dependent on the low frequency of $\mathrm{ER}-/ \mathrm{PgR}+$ phenotype. Thus, the conclusions from survival analyses should be interpreted with caution. In multiple cases, only biopsy material was available for analysis. Moreover, to save valuable tissue material (especially from core needle biopsies) only PgR 636- tumours were stained with the 1E2 clone. 


\section{CONCLUSIONS}

Pathologists should be cautious in every case of ER-/PgR+ tumour, especially when demonstrating low-grade morphology, low Ki67 index, or lack of internal ER control. Preferentially, re-evaluation should be performed with an alternative FFPE block, and, if possible, with the use of another antibody clone. In doubtful cases, mRNA assays, e.g., STRAT4, may be used to confirm the diagnosis. Further multicentre studies are necessary to establish the molecular landscape of these rare cancers, hopefully leading to the identification of new targets for personalised therapy.

Conflicts of interest and sources of funding: Elżbieta Senkus received honoraria and/or served advisory roles for Egis, Eli Lilly, Genomic Health, Novartis, Pfizer and Sandoz. Maria Litwiniuk received honoraria and/or served advisory roles for Pfizer, Roche, Novartis, Eli Lilly, Teva, AstraZeneca. Katarzyna Pogoda received honoraria and/or served advisory roles for Roche, AstraZeneca, Novartis, Eli Lilly, Pfizer, Teva. Joanna Pikiel received honoraria and/or served advisory roles for Novartis, Eli Lilly, Roche, MSD, AstraZeneca, GlaxoSmithKline, Regeneron, Pfizer. Aleksandra Łacko received honoraria and/or served advisory roles for Roche, AstraZeneca, Novartis, Eli Lilly, GlaxoSmithKline, Pfizer, Mylan, Exact Sciences, Teva. Barbara Radecka received honoraria and/or served advisor roles for BristolMyers Squibb, Lilly, Merck, MSD, Novartis, Pfizer, Pierre Fabre, Roche, Servier. These companies did not provide funding for this work. The other authors declare no conflict of interest. This research was funded by the National Science Centre, Poland (grant 2017/25/B/NZ5/00656).

Address for correspondence: Michał Kunc, MD, Department of Pathomorphology, Medical University of Gdańsk, Mariana Smoluchowskiego 17, 80-214 Gdansk, Poland. E-mail: mkunc@ gumed.edu.pl

Address for correspondence: Elżbieta Senkus, MD, PhD, Department of Oncology and Radiotherapy, Medical University of Gdańsk, Mariana Smoluchowskiego 17, 80-214 Gdansk, Poland. E-mail: elsenkus@gumed. edu.pl

\section{References}

1. Hefti MM, Hu R, Knoblauch NW, et al. Estrogen receptor negative/ progesterone receptor positive breast cancer is not a reproducible subtype. Breast Cancer Res 2013; 15: R68.

2. Nadji M, Gomez-Fernandez C, Ganjei-Azar P, Morales AR. Immunohistochemistry of estrogen and progesterone receptors reconsidered: experience with 5,993 breast cancers. Am J Clin Pathol 2005; 123 $21-7$

3. Maleki Z, Shariat S, Mokri M, Atri M. ER-negative/PR-positive breast carcinomas or technical artifacts in immunohistochemistry? Arch Iran Med 2012; 15: 366-9.

4. De Maeyer L, Van Limbergen E, De Nys K, et al. Does estrogen receptor-negative/progesterone receptor-positive breast carcinoma exist? $J$ Clin Oncol 2008; 26: 335-6.

5. Kunc M, Biernat W, Senkus-Konefka E. Estrogen receptor-negative progesterone receptor-positive breast cancer - "Nobody's land" or just an artifact? Cancer Treat Rev 2018; 67: 78-87.

6. Allison KH, Hammond MEH, Dowsett M, et al. Estrogen and progesterone receptor testing in breast cancer: ASCO/CAP guideline update. $J$ Clin Oncol 2020; 38: 1346-66.

7. Beltjens F, Molly D, Bertaut A, et al. ER-/PR+ breast cancer: a distinct entity, which is morphologically and molecularly close to triple-negative breast cancer. Int J Cancer 2021; 149: 200-13.
8. Fan Y, Ding X, Xu B, et al. Prognostic significance of single progesterone receptor positivity: a comparison study of estrogen receptor negative/progesterone receptor positive/Her2 negative primary breast cancer with triple negative breast cancer. Medicine 2015; 94: e2066.

9. Yu K, Di G, Wu J, et al. Breast cancer patients with estrogen receptornegative/progesterone receptor-positive tumors: being younger and getting less benefit from adjuvant tamoxifen treatment. $J$ Cancer Res Clin Oncol 2008; 134: 1347-54.

10. Giuliano AE, Edge SB, Hortobagyi GN. Eighth edition of the AJCC cancer staging manual: breast cancer. Ann Surg Oncol 2018; 25: $1783-5$.

11. Ahmed SS, Thike AA, Zhang K, Lim JCT, Tan PH. Clinicopathological characteristics of oestrogen receptor negative, progesterone receptor positive breast cancers: re-evaluating subsets within this group. J Clin Pathol 2017; 70: 320-6.

12. Cserni G, Francz M, Kálmán E, et al. Estrogen receptor negative and progesterone receptor positive breast carcinomas-how frequent are they? Pathol Oncol Res 2011; 17: 663-8.

13. Kuroda H, Muroi N, Hayashi M, et al. Oestrogen receptor-negative/ progesterone receptor-positive phenotype of invasive breast carcinoma in Japan: re-evaluated using immunohistochemical staining. Breast Cancer 2019; 26: 249-54.

14. Kornaga EN, Klimowicz AC, Guggisberg N, et al. A systematic comparison of three commercial estrogen receptor assays in a single clinical outcome breast cancer cohort. Mod Pathol 2016; 29: 799-809.

15. R Core Team. R: A Language and Environment for Statistical Computing. Vienna: R Foundation for Statistical Computing, 2020.

16. Wickham H. ggplot2: Elegant Graphics for Data Analysis. New York: Springer-Verlag, 2016.

17. Kassambara A, Kosinski M, Biecek P. survminer: drawing survival curves using "ggplot2". R package version 0.4.8. 25 Jul 2020; cited Aug 2021. https://cran.r-project.org/web/packages/survminer

18. Xiao N. ggsci: scientific journal and sci-fi themed color palettes for "ggplot2". 14 May 2018; cited Aug 2021. https://CRAN.R-project.org/ package $=$ ggsci

19. Dunnwald LK, Rossing M, Li CI. Hormone receptor status, tumor characteristics, and prognosis: a prospective cohort of breast cancer patients. Breast Cancer Res 2007; 9: R6.

20. Li Y, Yang D, Yin X, et al. Clinicopathological characteristics and breast cancer-specific survival of patients with single hormone receptorpositive breast cancer. JAMA Netw Open 2020; 3: e1918160.

21. Zhao H, Gong Y. The prognosis of single hormone receptor-positive breast cancer stratified by HER2 status. Front Oncol 2021; 11 643956.

22. Yu K-D, Jiang Y-Z, Hao S, Shao Z-M. Molecular essence and endocrine responsiveness of estrogen receptor-negative, progesterone receptor positive, and HER2-negative breast cancer. BMC Med 2015; 13: 254

23. Hopp TA, Weiss HL, Hilsenbeck SG, et al. Breast cancer patients with progesterone receptor PR-A-rich tumors have poorer disease-free survival rates. Clin Cancer Res 2004; 10: 2751-60.

24. McGowan EM, Clarke CL. Effect of overexpression of progesterone receptor A on endogenous progestin-sensitive endpoints in breast cancer cells. Mol Endocrinol 1999; 13: 1657-71.

25. Özgüzer A, Ertan Özgüzer G. The smallest subtype in the SEER Database: estrogen receptor negative progesterone receptor positive breast cancer. World Cancer Res J 2021; 8: e1848.

26. Brock JE, Hornick JL, Richardson AL, Dillon DA, Lester SC. A comparison of estrogen receptor SP1 and 1D5 monoclonal antibodies in routine clinical use reveals similar staining results. Am J Clin Pathol 2009; 132: 396-401.

27. Bogina G, Zamboni G, Sapino A, et al. Comparison of anti-estrogen receptor antibodies SP1, 6F11, and 1D5 in breast cancer. Am J Clin Pathol 2012; 138: 697-702.

28. Diorio C, Laberge S, Caron C, Provencher L, Hogue J-C, Sanschagrin F. Validation of EP1 antibody clone for estrogen receptor immunohistochemistry in breast cancer. Appl Immunohistochem Mol Morphol 2014; 22: $613-8$.

29. Badve S, Vladislav IT, Spaulding B, et al. EP1: a novel rabbit monoclonal antibody for detection of oestrogen receptor $\alpha$. J Clin Patho 2013; 66: $1051-7$.

30. Troxell ML, Long T, Hornick JL, Ambaye AB, Jensen KC. Comparison of estrogen and progesterone receptor antibody reagents using proficiency testing data. Arch Pathol Lab Med 2017; 141: 1402-12.

31. Kornaga EN, Klimowicz AC, Guggisberg N, et al. Evaluation of three commercial progesterone receptor assays in a single tamoxifen-treated breast cancer cohort. Mod Pathol 2016; 29: 1492-500. 